in the case of algebraic closure there is a stronger result of Hollkott (Hamburg): the axiom of choice is sufficient for the existence and uniqueness of algebraic closure.

An essential simplification is made possible for Baer's $\dagger$ theory of the degree of algebraic extensions. I plan to show elsewhere how the generalized continuum hypothesis may be avoided.

Yale University

\title{
CONCERNING TWO INTERNAL PROPERTIES OF PLANE CONTINUA
}

BY R. E. BASYE

Theorem 1 below was suggested to me by R. L. Moore. Theorem 2 is an extension of Kuratowski's result $\$$ that if three compact plane continua have a point in common and their sum separates a point $A$ from a point $B$ in the plane, then there exists a pair of these continua whose sum separates $A$ from $B$ in the plane. Another extension of this result along combinatorial lines has been given\| by Cech.

TheORem 1. Let $H$ and $K$ be two mutually exclusive and closed subsets of a compact continuum $M$ which lies in the plane. If for each pair of points $A$ and $B$ in $H$ and $K$, respectively, there exists a finite collection $\Gamma_{A B}$ of continua in $M$ such that $\Gamma_{A B}^{*}$ separates $A$ from $B$ in $M$, then there exists a finite collection $\Gamma$ of continua in $M$ such that $\Gamma^{*}$ separates $H$ from $K$ in $M$.

Let $\epsilon_{1}, \epsilon_{2}, \cdots$, be a sequence of positive numbers converging monotonically to zero, with $\epsilon_{1}$ less than half the distance from $H$ to $K$. For each $i$ let $D_{H}^{i}$ be a domain containing $H$ such that (1) the boundary $\beta_{H}^{i}$ of $D_{H}^{i}$ is the sum of a finite number of mutually exclusive simple closed curves, and (2) each point of

$\dagger$ Eine Anwendung der Kontinuumhypothese in der Algebra. Journal für Mathematik, vol. 162.

$\ddagger$ Presented to the Society, April 6, 1935, under a somewhat different title.

$\S$ Kuratowski, Théorème sur trois continus, Monatshefte für Mathematik und Physik, vol. 36 (1929), pp. 77-80.

$\|$ E. Čech, Trois thêorèmes sur l'homologie, Publications de la Faculté des Sciences de L'Université Masaryk, No. 144, 1931, pp. 1-21. 
$D_{H}^{i}$ lies at a distance less than $\epsilon_{i}$ from $H$. For each $i$ let $D_{K}^{i}$ be a similar domain containing $K$ and having boundary $\beta_{K}^{i}$. We shall show that there exists a value of $i$ such that the number of components of $M-M \cdot\left(D_{H}^{i}+D_{K}^{i}\right)$ which intersect both $\beta_{H}^{i}$ and $\beta_{K}^{i}$ is finite.

Suppose there are infinitely many such components for each value of $i$. Consider for each $i$ an infinite sequence $C_{1}{ }^{i}, C_{2}{ }^{i}, \ldots$, of components of $M-M \cdot\left(\overline{D_{H}^{i}}+\overline{D_{K}^{i}}\right)$, no two of which lie in the same component of $M-M \cdot\left(D_{H}^{i}+D_{K}^{i}\right)$, and such that both $\beta_{H}^{i}$ and $\beta_{K}^{i}$ contain a limit point of $C_{j}^{i}(j=1,2, \cdots)$. There exists an infinite subsequence $\alpha^{i}=E_{1}{ }^{i}, E_{2}{ }^{i}, \cdots$ of the sequence $\overline{C_{1}}, \overline{C_{2}}, \cdots$, and two components $j_{H}^{i}, j_{K}^{i}$ of $\beta_{H}^{i}, \beta_{K}^{i}$, respectively, such that each element of $\alpha^{i}$ intersects both $j_{H}^{i}$ and $j_{K}^{i}$.

No component of $\beta_{H}^{i}$ or $\beta_{K}^{i}$ distinct from $j_{H}^{i}$ and $j_{K}^{i}$ can intersect more than two elements of $\alpha^{i}$. For suppose $j$ is such a component, intersecting, say, the three elements $E_{1}^{i}, E_{2}^{i}, E_{3}{ }^{i}$ of $\alpha^{i}$. The continua $E_{1}{ }^{i}, E_{2}{ }^{i}, E_{3}{ }^{i}$ are mutually exclusive, intersect $j$, and are not disconnected by $j$. It follows that no two points of $j_{H}^{i} \cdot E_{n}^{i},(n=1,2,3)$, are separated from each other in $j_{H}^{i}$ by $\sum_{r=1}^{3} E_{r}^{i}-E_{n}^{i}$. Hence there exist three $\operatorname{arcs} b_{1}, b_{2}, b_{3}$ such that $(1) b_{1}+b_{2}+b_{3}=j_{H}^{i}$, and (2) $b_{n},(n=1,2,3)$, contains $j_{H}^{i} \cdot E_{n}^{i}$ but no point of $\sum_{r=1}^{3} E_{r}^{i}-E_{n}^{i}$. Denote by $F_{n}^{i},(n=1,2,3)$, the continuum $E_{n}^{i}+b_{n}+j$. Let $\delta_{H}^{i}$ and $\delta_{K}^{i}$ denote the complementary domains of $j_{H}^{i}$ and $j_{K}^{i}$ which do not contain $j_{K}^{i}$ and $j_{H}^{i}$, respectively. The three continua $F_{n}{ }^{i},(n=1,2,3)$, have a point in common and their sum separates a point $X$ of $\delta_{H}^{i}$ from a point $Y$ of $\delta_{K}^{i}$ in the plane. Hence, by a theorem of Kuratowski, $\dagger$ there exist two of these continua, say $F_{1}{ }^{i}$ and $F_{2}{ }^{i}$, such that $F_{1}{ }^{i}+F_{2}^{i}$ separates $X$ from $Y$ in the plane. But $\delta_{H}{ }^{i}+\left(E_{3}{ }^{i}-j \cdot E_{3}{ }^{i}\right)+\delta_{K}{ }^{i}$ is a connected set which contains $X$ and $Y$ but no point of $F_{1}^{i}+F_{2}^{i}$.

Consequently, the number of components of $\beta_{H}^{i}+\beta_{K}^{i}$ being finite, there exists an integer $r$ such that each element of the sequence $\gamma^{i}=E_{r}^{i}, E_{r+1}^{i}, \cdots$, intersects both components $j_{H}^{i}$, $j_{K}^{i}$ without meeting any other component of $\beta_{H}^{i}+\beta_{K}^{i}$. The intersection of each element $E_{n}^{i}$ of $\gamma^{i}$ with $j_{H}^{i}$ is contained in an arc (or point) of $j_{H}^{i}$ which intersects no other element of $\gamma^{i}$. Denote by $a_{n}{ }^{i}$ the minimal such arc (or point), and by $b_{n}{ }^{i}$ a

† Loc. cit. 
similar arc (or point) containing the intersection of $E_{n}^{i}$ with $j_{K}{ }^{i}$. The sequence $\left\{a_{n}{ }^{i}+b_{n}{ }^{i}\right\},(n=r, r+1, \cdots)$, contains a subsequence which has a sequential limiting set; let $P_{i}$ and $Q_{i}$ denote points of this limiting set which lie on $j_{H}^{i}$ and $j_{K}^{i}$ respectively. The sequence $\left\{P_{i}+Q_{i}\right\}$ contains a subsequence which has a sequential limiting set; let $P$ and $Q$ denote points of this limiting set which lie in $H$ and $K$, respectively.

By hypothesis there exists a finite collection $\Gamma_{P Q}$ of continua in $M$ such that $\Gamma_{P Q}^{*}$ separates $P$ from $Q$ in $M$. Hence $M-\Gamma_{P Q}^{*}$ $=N_{P}+N_{Q}$, where $N_{P}$ and $N_{Q}$ are mutually separated sets containing $P$ and $Q$, respectively. Let $R_{P}$ and $R_{Q}$ be regions containing $P$ and $Q$, respectively, such that $R_{P} \cdot\left(N_{Q}+\Gamma_{P Q}^{*}\right)=R_{Q} \cdot\left(N_{P}\right.$ $\left.+\Gamma_{P Q}^{*}\right)=0$. Let $i$ be chosen so that $P_{i}$ and $Q_{i}$ lie in $R_{P}$ and $R_{Q}$, respectively. Then there exists an infinite subsequence $\epsilon^{i}=E_{n, 1}^{i}$, $E_{n, 2}^{i}, \cdots$ of $\gamma^{i}$ such that $a_{n, t}^{i}$ and $b_{n, t}^{i},(t=1,2, \cdots)$, are subsets of $R_{P}$ and $R_{Q}$, respectively. Every element of $\epsilon^{i}$ intersects some element of $\Gamma_{P Q}$. Therefore some element $W$ of $\Gamma_{P Q}$ intersects at least two elements $\epsilon^{i}$, hence contains a point of $\beta_{H}^{i}+\beta_{K}^{i}$. Hence, if $E_{n, s}^{i}$ denotes an element of $\epsilon^{i}$ which intersects $W$, then $W$ contains a point of $a_{n, 8}^{i}+b_{n, s}^{i}$. But $a_{n, s}^{i}+b_{n, s}^{i}$ is a subset of $R_{P}+R_{Q}$, which contains no point of $\Gamma_{P Q}^{*}$.

It follows that there exists a value of $i$ such that the collection $\Gamma$ of components of $M-M \cdot\left(D_{H}^{i}+D_{K}^{i}\right)$ which intersect both $\beta_{H}^{i}$ and $\beta_{K}^{i}$ is finite. Denote by $U_{H}$ the sum of those components of $M-M \cdot\left(D_{H}^{i}+D_{K}^{i}\right)$ which intersect $\beta_{H}^{i}$ but not $\beta_{K}^{i}$, and by $U_{K}$ the sum of those components which intersect $\beta_{K}^{i}$ but not $\beta_{H}^{i}$. It can be seen that $M \cdot D_{H}^{i}+U_{H}$ and $M \cdot D_{K}^{i}+U_{K}$ are mutually separated sets containing $H$ and $K$, respectively. Hence $\Gamma^{*}$ separates $H$ from $K$ in $M$.

R. L. Moore has proved $\dagger$ the closely related result whose statement is identical with that of Theorem 1 except that each $\Gamma_{A B}$ and $\Gamma$ are taken as finite sets. The argument is valid in any space satisfying his axioms 0 and 1 . An example showing that Theorem 1 is not true for three-dimensional continua has been given by W. T. Reid.

The conclusion of Theorem 1 will also hold if $\Gamma_{A B}^{*}$ merely

$\dagger$ Foundations of Point Set Theory, Colloquium Publications of this Society, vol. 13, p. 140, Theorem 57.

$\ddagger$ See this issue of this Bulletin, pp. 683-684. 
weakly disconnects $\dagger A$ from $B$ in $M$, provided $M$ is locally connected at each point of $H+K$. The argument is for the most part similar.

Again, if we require only that $\Gamma_{A B}$ be a countable collection of continua in $M$ such that $\Gamma_{A B}^{*}$ is closed and separates $A$ from $B$ in $M$, then there exists a countable collection $\Gamma$ of continua in $M$ such that $\Gamma^{*}$ is closed and separates $H$ from $K$ in $M$.

TheOREм 2. Let $M$ be any subcontinuum of a plane or a sphere and let $Z$ be any subset (possibly vacuous) of $M$. If $G$ is a finite collection of connected subsets of $M$ such that (1) there exist $r$ points ( $r$ finite) whose sum $L$ intersects each element of $G$, and (2) $G^{*}+Z$ weakly disconnects a point $A$ from a point $B$ in $M$, then there exists a subcollection $H$ of $G$, containing not more than $2 r$ elements, such that $H^{*}+Z$ weakly disconnects $A$ from $B$ in $M$.

The case where $M$ is a subset of a plane is a consequence of the case where $M$ is a subset of a sphere. Suppose, then, that $M$ is a subcontinuum of a sphere $S$. We shall discuss in order the three cases that may arise.

Case 1. Suppose $r=1$. Assume the theorem false. Let $g_{1}, \cdots$, $g_{n}$ denote the elements of $G$ and let $O$ be a point common to these elements. There exist continua $C_{i j},(i, j=1, \cdots, n)$, such that $C_{i j}$ contains $A+B$ and has no point in common with $g_{i}+g_{j}+Z$. Let $D_{k},(k=1, \cdots, n)$, be a connected domain which contains $g_{k}$ and contains no point of any $C_{k j},(j=1, \cdots, n)$. Let $D_{0}$ be a domain which contains $Z$ but contains no point of any $C_{i j},(i, j=1, \cdots, n)$. Let $\Delta=\sum_{i=0}^{n} D_{i}$. The domain $(S-M)+\Delta$ weakly disconnects, hence separates, $A$ from $B$ in $S$. Hence there exists a component $Q$ of $(S-M)+\Delta$ which separates $A$ from $B$ in $S$, and $Q$ must contain the connected set $\sum_{i=1}^{n} D_{i}$.

If $Q_{i},(i=1, \cdots, n)$, denotes that component of $(S-M)$ $+D_{0}+D_{i}$ which contains $g_{i}$, then $Q=Q_{1}+\cdots+Q_{n}$. For suppose the contrary and let $R$ denote the set $Q-\left(Q_{1}+\cdots\right.$ $\left.+Q_{n}\right)$. Since $Q_{1}+R$ is not connected, $R$ contains a subset $R_{1}$ such that $R_{1}$ and $\left(Q_{1}+R\right)-R_{1}$ are mutually separated sets. In general, if $R_{i}$ has been defined, $R_{i+1},(i=1, \cdots, n-1)$, will

$\dagger A$ set $L$ weakly disconnects a set $A$ from a set $B$ in a connected set $M$ if it contains a point of every connected and relatively closed subset of $M$ which intersects both $A$ and $B$. 
denote a subset of $R_{i}$ such that $R_{i+1}$ and $\left(Q_{i+1}+R_{i}\right)-R_{i+1}$ are mutually separated sets. It follows that $R_{n}$ and $Q-R_{n}$ are mutually separated sets, contrary to the connectedness of $Q$.

Let $F_{i t},(i=1, \cdots, n ; t=1,2, \cdots)$, be a subcontinuum of $Q_{i}$ which contains $O$ and every point of $Q_{i}$ whose distance from the boundary of $Q_{i}$ is greater than $1 / t$. Let $F_{t}=F_{1 t}+\cdots+F_{n t}$, $(t=1,2, \cdots)$. There exists a value $w$ of $t$ such that $F_{w}$ separates $A$ from $B$ in $S$. For on the contrary supposition there exists a sequence of continua $N_{1}, N_{2}, \cdots$, such that $N_{t},(t=1,2, \cdots)$, contains $A+B$ and contains no point of $F_{t}$. The limiting set of this sequence is a continuum which contains $A+B$ but no point of $Q_{1}+\cdots+Q_{n}$. But this is impossible. Consequently $\dagger$ there exist two of the continua $F_{i w}$, say $F_{1 w}$ and $F_{2 w}$, whose sum separates $A$ from $B$ in $S$. Hence $Q_{1}+Q_{2}$ separates $A$ from $B$ in $S$. But the continuum $C_{12}$ contains $A+B$ and has no point in common with $Q_{1}+Q_{2}$.

CASE 2. Suppose that $r>1$ and $G^{*}$ is connected. There exists a subcollection $K$ of $G$ having not more than $2 r-2$ elements and such that $K^{*}$ is a connected set which contains $L$. Denote by $G^{\prime}$ the collection of all elements each of which is the sum of $K^{*}$ and an element of $G$. The elements of $G^{\prime}$ are connected sets having $K^{*}$ in common; hence, by Case 1 , there exist two elements $g_{1}^{\prime}$ and $g_{2}^{\prime}$ of $G^{\prime}$ such that $g_{1}^{\prime}+g_{2}^{\prime}+Z$ weakly disconnects $A$ from $B$ in $M$. The set $g_{1}^{\prime}+g_{2}^{\prime}$ is the sum of $2 r$ or less elements of G.

CASE 3. Suppose merely that $r>1$. Let $H$ be a subcollection of $G$ which is irreducible with respect to the property that $H^{*}+Z$ weakly disconnects $A$ from $B$ in $M$. Let $C_{1}, \cdots, C_{q}$ denote the components of $H^{*}$ and let $r_{i},(i=1, \cdots, q)$, be the number of points common to $L$ and $C_{i}$. By Case 2 there exist $2 r_{i}$ or less elements of $H$ lying in $C_{i},(i=1, \cdots, q)$, such that, if $T_{i}$ denotes their sum, $T_{i}+\left[Z+\left(H^{*}-C_{i}\right)\right]$ weakly disconnects $A$ from $B$ in $M$. Hence $C_{i}$ cannot contain more than $2 r_{i}$ elements of $H$. Therefore, since $r \geqq r_{1}+\cdots+r_{q}, H^{*}$ cannot contain more than $2 r$ elements of $G$.

The University of TeXas

† C. Kuratowski, loc. cit. 